\title{
Vacuum infusion of a composite E-glass vinylester laminate for nautical application: experimental response to repeated impacts
}

\author{
G. Belingardi, M. P. Cavatorta \& D. S. Paolino \\ Department of Mechanical Engineering, Politecnico di Torino, Italy
}

\begin{abstract}
The aim of this paper is to investigate the response to repeated impacts of a non-symmetric glass fibre reinforced laminate. The laminate is intended for a nautical application and the manufacturing technique is vacuum infusion. In a first part of the work, four impact velocities $(1.566 \mathrm{~m} / \mathrm{s}, 2.215 \mathrm{~m} / \mathrm{s}, 3.132 \mathrm{~m} / \mathrm{s}$ and $3.836 \mathrm{~m} / \mathrm{s}$ ) were considered, and a minimum of four specimens for any given velocity were subjected to forty repeated impacts or up to perforation. The impact response was evaluated in terms of damage progression by visual observation of the impacted specimens, evolution of the peak force and of the bending stiffness with the number of impacts and by calculating the damage degree (ratio between the absorbed energy and the impact energy). In a second part of the work, additional single impact tests were performed at $4.429 \mathrm{~m} / \mathrm{s}$ and $6.264 \mathrm{~m} / \mathrm{s}$ to investigate the laminate strain-rate sensitivity.

Repeated impact tests pointed out that the delamination area grows very rapidly in the first few impacts to then level off, following closely the behaviour of the bending stiffness against the number of impacts. The value of the peak force increases in the first few impacts due to compaction and strain-hardening of the resin with a related decrease of the damage degree. Comparison between quasi-static penetration tests and impact tests demonstrates a strain-rate dependency of the laminate bending stiffness and first damage load. The values of $\mathrm{R}^{2}$ show a good fitness of a linear function on the experimental data.

Keywords: repeated impacts, strain rate, E-glass vinylester laminates, vacuum infusion.
\end{abstract}




\section{Introduction}

Vacuum infusion is a manufacturing technique that uses vacuum pressure to drive resin into a laminate. Materials are laid dry into the mould and the vacuum is applied before the resin is introduced. Once a complete vacuum is achieved, resin is literally sucked into the laminate via carefully placed tubing. In a typical hand lay-up, reinforcements are laid into the mould and manually wet out using brushes or rollers. Because of this, the laminate starts in an oversaturated state. Vacuum bagging will remove much of the resin in excess but the amount removed will still depend on a variety of variables including type of reinforcement, resin and time factors. Vacuum infusion takes a different approach, in that a vacuum is drawn while the materials are still dry. From that point, resin is infused using vacuum pressure. Rather than starting with an excess of resin, vacuum infusion starts with none and pushes resin in. Ideally, any excess of resin that is introduced will be sucked out into the vacuum line. Thus, parts constructed by vacuum infusion are said to approach prepreg levels of resin content. Another benefit of vacuum infusion is the lack of time constraints due to the pot-life of the resin which can be crucial with large products. Finally, vacuum infusion is a much cleaner and friendlier work environment process as the inhaling of resin fumes by operators is reduced to a minimum. Because of these potential benefits, vacuum infusion is being considered as a viable alternative to more traditional hand lay-up despite of the higher costs and longer set-up times.

In the present paper a glass fibre reinforced plastics laminate intended for nautical application and manufactured by vacuum infusion is tested to repeated impacts. The loading condition of repeated impacts is of particular relevance in the naval or nautical fields. Low velocity impacts on laminates are known to significantly reduce the laminate strength and stiffness, mainly as a consequence of multiple stacked delaminations that are produced by the impact loading at a number of interfaces through the thickness of the composite laminate. For composites, the damage induced by impact loading is more subtle than in metals, as it is often not detectable, beginning on the non-impacted surface or in the form of internal delamination [1]. SEM observations showed that, even without any visible external damage, microcracks in the resin can produce internal delaminations, thus reducing the composite laminate strength [2].

Four falling heights are considered, corresponding to conditions of no perforation within test duration and to conditions of laminate perforation. The impact response is evaluated in terms of damage evolution by visual observation of the impacted specimens, evolution of the peak force with the number of impacts, stiffness reduction against number of impacts, and by calculating the damage degree according to Belingardi et al. [3] and Belingardi and Vadori [4]. The damage degree is defined as the ratio between the absorbed energy and the impact energy. In the case of a rebound, the damage degree is below one. In the case the impactor is stopped with no rebound or perforation or when specimen perforation occurs, the damage degree reaches the value of one. 
Comparison between quasi-static penetration tests and dynamic tests at different impact velocities is taken to investigate the laminate strain-rate sensitivity. The literature on the strain-rate sensitivity of fibre reinforced composites is not straightforward. While it is generally accepted that the mechanical properties of most polymer matrix composites are rate sensitive, literature results are often contradictory as shown by Barré et al. [5], summarising test data from 31 papers, and very recently by Wang et al. [6]. Although inconsistencies maybe due to differences in matrix, reinforcement alignment and test conditions, they quite imply the complex nature of rate dependency for polymer matrix composites [7].

\section{Experimental testing}

The laminate under study is a glass fibre reinforced plastics. The stacking sequence is reported in Table 1. Two resin systems are used: vinylester and polyester. Main reason for the asymmetry of the laminate, in both the resin system and the stacking sequence, is cost reduction. The interface between the two resins is located at about two thirds of the laminate thickness from the vinylester side. The fibre reinforcement varies from $35 \%$ in weight in the mat, $45 \%$ in the bidirectional lamina (BD) and 50\% in the unidirectional lamina (UD).

Table 1: $\quad$ Stacking sequence of the laminate.

\begin{tabular}{|c|c|c|c|}
\hline & ply type & $\mathrm{t}(\mathrm{mm})$ & N. of plies \\
\hline \multirow{4}{*}{ vinylester } & mat & 0.74 & 2 \\
\hline & UD & 1.25 & 2 \\
\hline & $\mathrm{BD} 0 / 90$ & 1.40 & 2 \\
\hline & mat & 0.74 & 1 \\
\hline \multirow{4}{*}{ polyester } & $\mathrm{BD} 0 / 90$ & 1.40 & 2 \\
\hline & UD & 1.25 & 1 \\
\hline & mat & 0.74 & 1 \\
\hline & total t (mm) & 12.31 & \\
\hline
\end{tabular}

Specimens were impacted on the polyester face of the laminate. Previous tests [8] have indeed shown that, while higher peak forces are achieved by impacting the vinylester face, the number of impacts to perforation is greater when impacting the polyester side of the laminate. The experimental result was explained by considering that, in the case it is the polyester face to be impacted, the more ductile resin -vinylester- is on the non-impacted face of the laminate where delamination is known to initiate [2], thus limiting the progression of damage. Moreover, it was observed that the condition of laminate perforation is associated with the splitting of laminae, which always occurs at the interface between the two resin systems. Therefore perforation is delayed in the case the 
impacted face is polyester, being the interface between the two resin systems more distant from the non-impacted face.

For impact tests, four falling heights were considered $(125 \mathrm{~mm}, 250 \mathrm{~mm}$, $500 \mathrm{~mm}, 750 \mathrm{~mm})$ corresponding to four impact velocities $(1.566 \mathrm{~m} / \mathrm{s}$, $2.215 \mathrm{~m} / \mathrm{s}, 3.132 \mathrm{~m} / \mathrm{s}$ and $3.836 \mathrm{~m} / \mathrm{s}$ ) and a minimum of four specimens for any given velocity were subjected to forty repeated impacts or up to perforation. Experimental impact tests were performed according to ASTM 3029 standard [9] using an instrumented free-fall drop dart testing machine. The impactor has a total mass of $20 \mathrm{~kg}$, its head is hemispherical with a radius of $10 \mathrm{~mm}$. Stainless steel was chosen for its high hardness and resistance to corrosion. The maximum falling height of the testing machine is $2 \mathrm{~m}$, which corresponds to a maximum impact energy of $392.4 \mathrm{~J}$. The drop-weight apparatus was equipped with a motorized lifting track. The collected data were stored after each impact and the impactor was returned to its original starting height. Using this technique, the chosen impact velocity was consistently obtained in successive impacts. Because, the target holder was rigidly attached to the frame of the testing device, the tup struck the specimen each time at the same location. By means of a piezoelectric load cell, force-time curves were acquired and, with a double integration, force-displacement and deformation energy-displacement curves were obtained. Initial conditions were given with the time axis having its origin at the time of impact. At time $\mathrm{t}=0$, the dart coordinate is zero and its initial velocity can be obtained by the well known relationship:

$$
v_{0}=\sqrt{2 g \Delta h}
$$

where $\Delta \mathrm{h}$ is defined as the height loss of the centre of mass of the dart with respect to the reference surface [4]. The drop dart machine used in the study is equipped with an optoelectronic device for measurement of the impact velocity. Agreement between measured and theoretical values was very good.

Square specimen panels, with $100 \mathrm{~mm}$ edge, were clamped with a $76.2 \mathrm{~mm}$ inner diameter, and fixed to a rigid base to prevent slippage of the specimen. The clamping system was designed to provide an adequate uniform pressure all over the clamping area. To assess the progression of damage, for one series of repeated impact tests at any given impact velocity, pictures of the specimen impacted and non-impacted face as well as of the specimen thickness were taken after each impact. A software program was used to determine the area of delamination, readily seen under light due to a change in the opacity of the resin. In addition to visual observation of impacted specimens, for all tests the variation of bending stiffness against impact number was evaluated from the force-displacement curves.

Prior to impact tests, a series of static indentation tests were performed to get information on the material stiffness and strength characteristics, which serve as a starting point to decide on the falling heights of impact tests. For quasi-static penetration tests, specimens were tested using a servo-hydraulic machine with maximum loading capacity of $100 \mathrm{kN}$. The hydraulic actuator was electronically controlled in order to perform constant velocity tests. Signals of the force applied 
by the actuator and of the actuator stroke were acquired in time with an appropriate sampling rate.

\section{Results and discussion}

Figure 1 depicts the force-deflection curves for one series of repeated impact tests run at an impact velocity of $2.215 \mathrm{~m} / \mathrm{s}$. The group of curves shows that the laminate stiffness diminishes as the impact number increases and that the highest reduction is achieved in the first few impacts. The higher the impact velocity, the greater the total decrease in laminate stiffness (Figure 2).

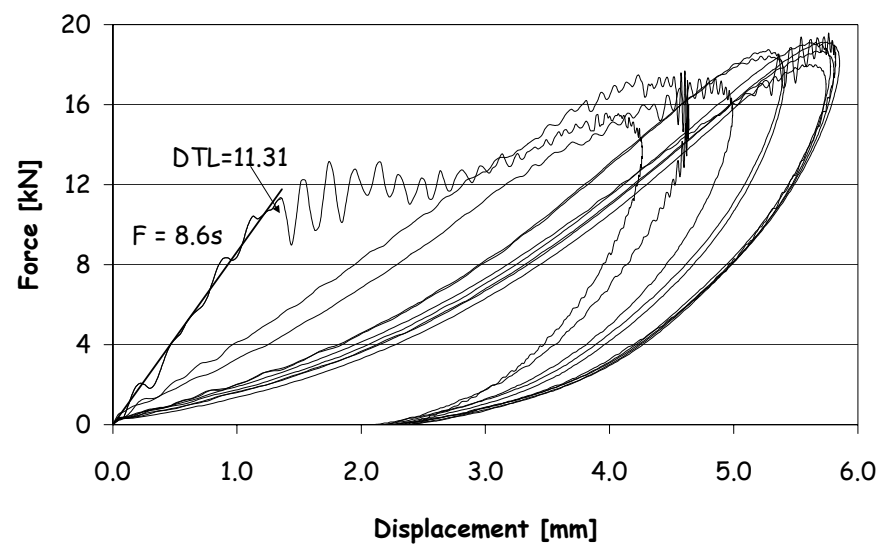

Figure 1: Representative force-deflection curves for repeated impact tests. Impact velocity: $2.215 \mathrm{~m} / \mathrm{s}$.

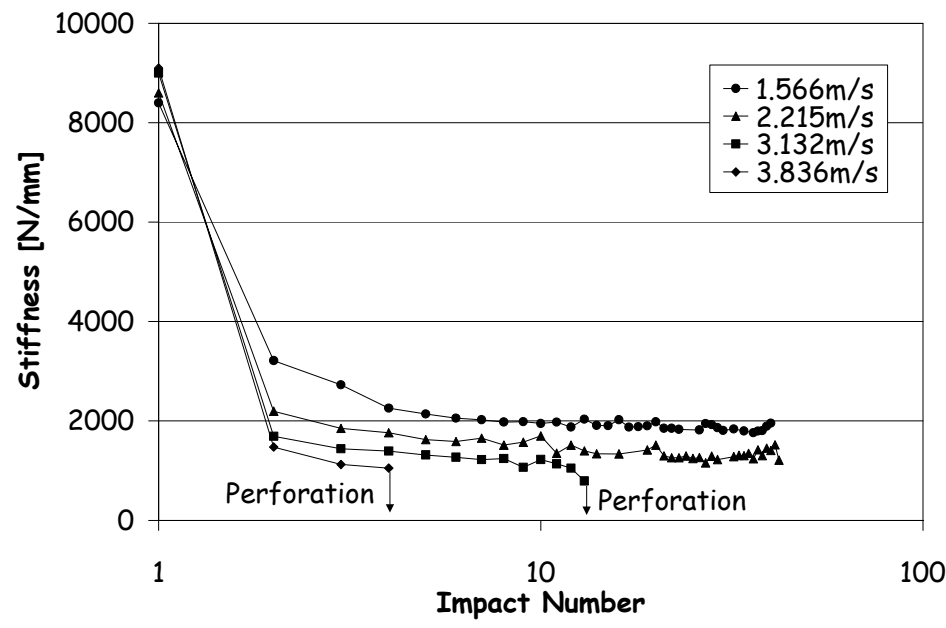

Figure 2: $\quad$ Loss of bending stiffness with impact loading. 


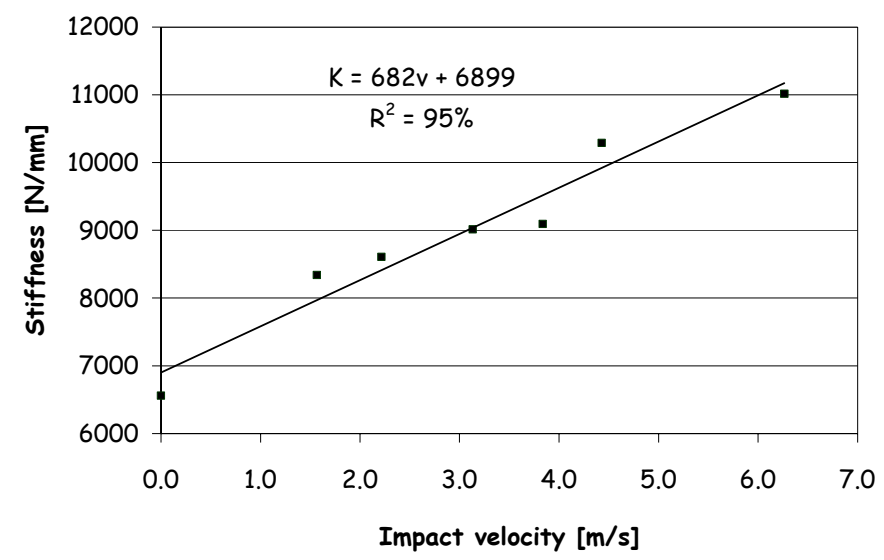

(a)

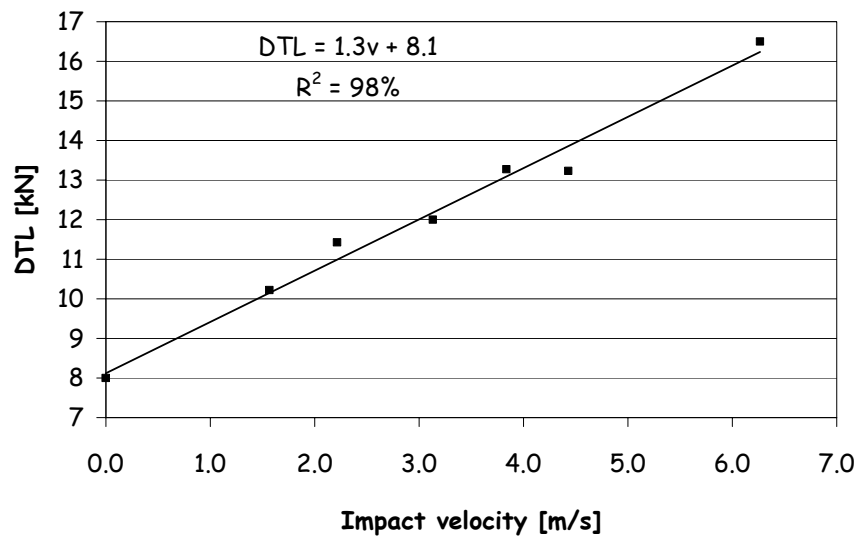

(b)

Figure 3: Laminate bending stiffness (a) and Delamination Threshold Load DTL- (b) as a function of impact velocity. Data points are mean values.

From Figure 2, it can be observed that the value of the bending stiffness, as evaluated from the initial portion of the force-displacement curve obtained in the first impact (Figure 1), is not constant at different impact velocities. In the attempt to investigate the strain-rate sensitivity and to widen the strain-rate range under study, additional single impact tests were performed at $4.429 \mathrm{~m} / \mathrm{s}$ and at $6.264 \mathrm{~m} / \mathrm{s}$, corresponding to the maximum height of the drop-dart apparatus. Figures 3 depict the laminate stiffness (Figure 3(a)) and the initial damage load in the form of the delamination threshold load (DTL) [10] (Figure 3(b)) as a function of impact velocity. Data points are mean values at any given impact velocity. Values obtained in quasi-static penetration tests are reported on the vertical axis. Since in impact tests no perforation was achieved in the first impact, no comparison of data could be carried out in terms of maximum load. Besides the range of impact velocities is rather limited, data plotted in Figures 3 
clearly demonstrate a strain-rate dependency of the stiffness and initial damage force. Values of $\mathrm{R}^{2}$ show a good fitness of a linear function on the experimental data.
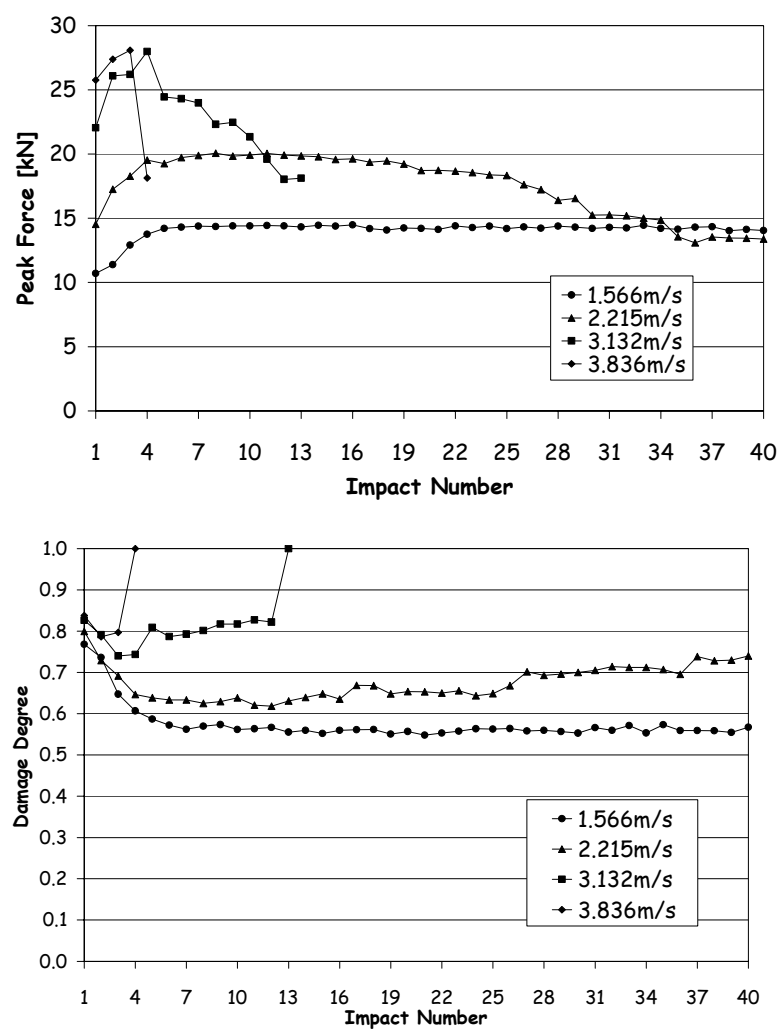

(a)

(b)

Figure 4: Peak force (a) and damage degree (b) as a function of impact number.

Figures 4 plot the peak force and the damage degree as a function of the number of impacts. It should be said that reported data are not average values but refer to one series of repeated impact tests. The choice was taken considering that for impact velocities that cause perforation, scattering in the peak force and damage degree values was observed in the one-two impacts before perforation. On the contrary, for velocities for which no perforation occurred within test duration, single test and average values agree very well. Data of Figures 4 show that for the two highest impact velocities, perforation of the laminate occurred in the fourth and thirteenth impact respectively, as clearly indicated by the damage degree that reaches the value of one. For the two lowest impact velocities, no perforation of the laminate was achieved within forty impacts. However, while for the $1.566 \mathrm{~m} / \mathrm{s}$ test both the peak force and the damage degree reach a stable 
value indicating the reaching of a steady-state condition, for the $2.215 \mathrm{~m} / \mathrm{s}$, the peak force continues to slowly decrease with increasing number of impacts, suggesting a slow but steady accumulation of damage. For one specimen at each impact velocity, the test was continued up to 60 impacts but no perforation was achieved for both specimens.

From Figure 4(a) it can also be observed that for all impact velocities, the maximum of the peak force is not reached in the first impact. According to [11], in repeated impact tests an initial increase in the peak force is possible due to the compaction process of the thin layer of unreinforced resin at the impacted surface. At low impact energy levels, damage to the fibres near the surface is minimal and the compaction process provides a harder surface for the next impact. In [12], the maximum in the peak force is interpreted as the turning point between indentation and local matrix cracking around the impacted region and major delamination of the impacted specimen.

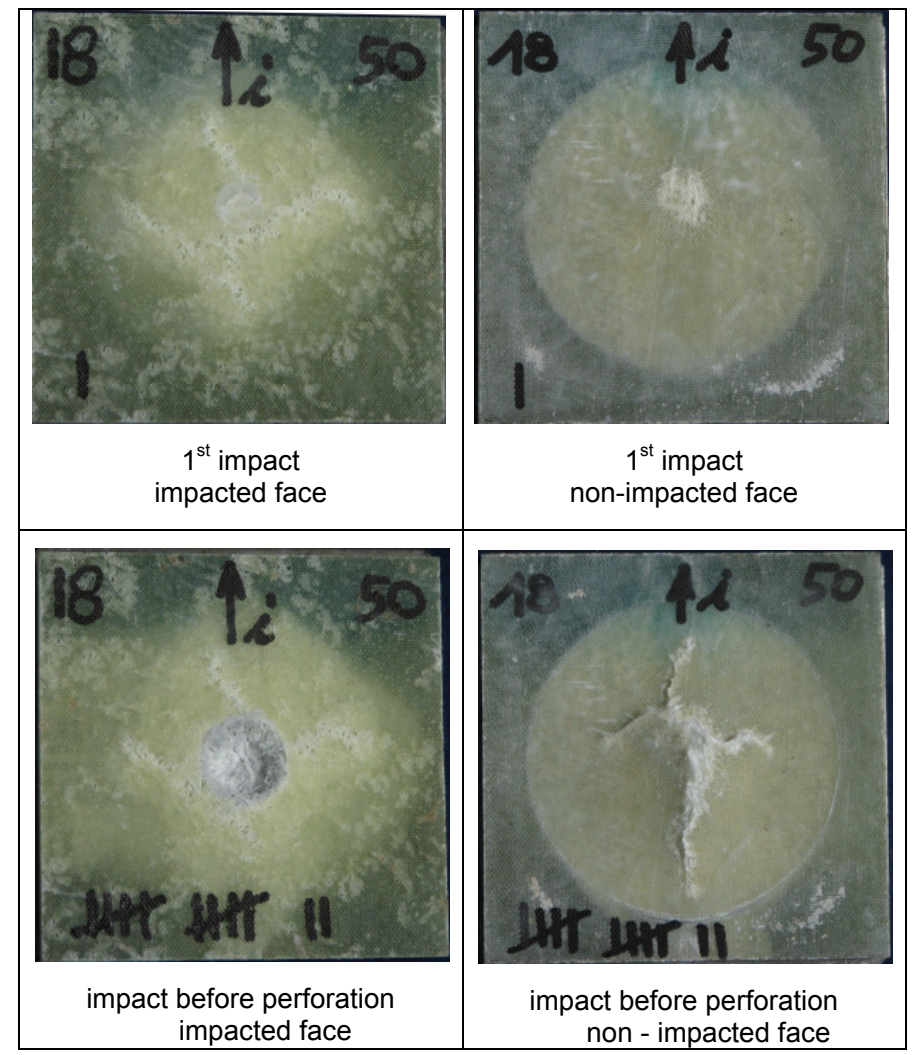

Figure 5: $\quad$ Pictures of impacted specimens. Impact velocity: $3.312 \mathrm{~m} / \mathrm{s}$.

When considering the curves depicted in Figure 4(a), the compaction process suggested in [11] may explain the reaching of a steady-state value in the peak 
force for the $1.566 \mathrm{~m} / \mathrm{s}$ tests as well as the initial rise in the peak force observed for the $2.215 \mathrm{~m} / \mathrm{s}$ tests. On the contrary, visual observation of the impacted specimens (Figure 5) seems not to support the findings reported in [12]. Indeed, for the impact velocities of $3.132 \mathrm{~m} / \mathrm{s}$ and $3.836 \mathrm{~m} / \mathrm{s}$, delamination appears to be the dominant damage mode from the very first impact. In the authors' opinion, it should be considered that the value of the peak force is affected by two opposite mechanisms: on one side the compaction and strain-hardening of the resin which provides a harder surface for the next impact event and on the other side the progression of damage which reduces the laminate mechanical properties. Initially, the beneficial local surface effect of resin compaction can determine an increase in the peak force, even if the global bending stiffness of the laminate has decreased.

Figure 4(b) plots the damage degree as a function of the impact number. As it can be expected, higher impact velocities are associated with higher damage degrees. The difference among impact velocities is rather limited in the first impact to become more and more important as the number of impacts increases. It is worthwhile noticing that there exists a correlation between the peak force and the damage degree diagrams. In particular, when the slope of the peak force plot is positive, the slope of the damage degree plot is negative. A maximum in the peak force is coupled with a minimum in the damage degree.

\section{Conclusions}

The paper summarises the experimental results of repeated impact tests performed on a non-symmetric glass fibre reinforced composite. The laminate is intended for a nautical application and the manufacturing technique is vacuum infusion. Four impact velocities were considered and a minimum of four specimens for any given velocity were subjected to forty repeated impacts or up to perforation. The impact response was evaluated in terms of damage progression by visual observation of the impacted specimens, evolution of the peak force and of the bending stiffness with the number of impacts and by calculating the damage degree (ratio between the absorbed energy and the impact energy). Single impact tests were also run at additional higher velocities to investigate the laminate strain-rate sensitivity. Based on observations made in this research work, the conclusions can be summarised as follows:

1. the bending stiffness and initial damage load of glass/vinylester composite laminates are rate sensitive, even at low range of strain rate. In particular, the relationship appears quite linear;

2. in repeated impact tests, the delamination area grows very rapidly in the first few impacts to then level off, following closely the behaviour of the bending stiffness against the number of impacts;

3. the value of the peak force increases in the first few impacts due to compaction and strain-hardening of the resin with a related decrease of the damage degree;

4. in the design and analysis of glass/vinylester composite structures, reference to data obtained in quasi-static penetration tests or at lower 
impact velocities seems to involve a good deal of safety considering that the greater the velocity and strain-rate, the higher the stiffness and initial damage load of the composite material.

\section{Acknowledgement}

The authors wish to acknowledge AZIMUT Yachts for supplying the specimens.

\section{References}

[1] Abrate S. Impact on composite structures. Cambridge University Press, 1998.

[2] Azouaoui K, Recha S, Azari Z, Benmedakhene S, Laksimi A, Pluvinage G, Modelling of damage and failure of glass/epoxy composite plates subject to impact fatigue. Int J Fatigue, 23, 877-885, 2001.

[3] Belingardi G, Grasso F, Vadori R, Energy absorption and damage degree in impact testing of composite materials. Proceedings XI ICEM (Int. Conf. Experimental Mechanics), Oxford (UK), 279-285, 1998.

[4] Belingardi G, Vadori R, Influence of the laminate thickness in low velocity impact behaviour of composite material plate. Composite Structures, 61, 27-38, 2003.

[5] Barrè $\mathrm{S}$, Chotard $\mathrm{T}$, Benzeggagh ML, Comparative study of strain rate effects on mechanical properties of glass fibre-reinforced thermoset matrix composites. Composites Part A, 27A, 1169-1181, 1996.

[6] Wang W, Makarov G, Shenoi RA, An analytical model for assessing strain rate sensitivity of unidirectional composite laminates. Composite Structures, 69, 45-54, 2005.

[7] Fereshteh-Saniee F, Majzoobi G.H., Bahrami M., An experimental study on the behavior of glass-epoxy composite at low strain-rates, Journal of Materials Processing Technology, 162, 39-45, 2005.

[8] Belingardi G, Cavatorta MP, Paolino DS, Repeated impact behaviour and damage progression of glass reinforced plastics. 16th European Conference of Fracture (ECF16), Alexandroupolis, Greece, July 3-7, 2006.

[9] ASTM D3029 - Standard Test Method for Impact Resistance of Rigid Plastic Sheeting or Parts by means of a Tup (Falling Weight). American Society for Testing Materials, 1982.

[10] Schoeppner GA, Abrate S, Delamination threshold loads for low velocity impact on composite laminate. Composites: Part A, 31(9), 903-915, 2000.

[11] Wyrick DA, Adams D F, Residual strength of a carbon/epoxy composite material subjected to repeated impact. J. Composite Materials, 22, 749-765, 1998.

[12] Liu D, Characterization of impact properties and damage process of glass/epoxy composite laminates. J. Composite Materials, 38, 1425-1442, 2004. 